\title{
Atomic Processes for Astrophysical Plasmas
}

\author{
N. R. Badnell ${ }^{1}$, G. Del Zanna ${ }^{2}$, L. Fernández-Menchero ${ }^{1}$, \\ A. S. Giunta ${ }^{3}$, G. Y. Liang ${ }^{4}$, H. E. Mason ${ }^{2}$ and P. J. Storey \\ ${ }^{1}$ Department of Physics, University of Strathclyde, Glasgow G4 0NG, UK \\ 2 DAMTP, Centre for Mathematical Sciences, University of Cambridge, \\ Wilberforce Road, Cambridge CB3 0WA, UK \\ ${ }^{3}$ STFC Rutherford Appleton Laboratory, Chilton, Didcot, OX11 0QX, UK \\ ${ }^{4}$ Key Laboratory of Optical Astronomy, National Astronomical Observatories, \\ Chinese Academy of Sciences, 100012 Beijing, PR China \\ ${ }^{5}$ Department of Physics and Astronomy, University College London, London \\ WC1E 6BT, UK
}

\begin{abstract}
In this review we summarize the recent calculations and improvements of atomic data that we have carried out for the analysis of astrophysical spectroscopy within the Atomic Processes for Astrophysical Plasmas (APAP) network. We briefly discuss the various methods used for the calculations, and highlight several issues that we have uncovered during such extensive work. We discuss the completeness and accuracy of the cross sections for ionic excitation by electron impact for the main isoelectronic sequences, which we have obtained with large-scale calculations. Given its astrophysical importance, we emphasize the work on iron. Some examples on the significant improvement that has been achieved over previous calculations are provided.
\end{abstract}




\section{Introduction}

High-resolution spectroscopy is now very common in a broad range of astrophysical research, using observations from both the ground and space across the whole spectrum, from the radio to hard X-rays. The sensitivity and resolution has increased so much that ever more accurate atomic data and complex modelling are required.

In this review we summarize the recent calculations and improvements of atomic data that we have carried out within the Atomic Processes for Astrophysical Plasmas (APAP) network. This work was funded by STFC (UK), and all the atomic data are made available via our website $\ddagger$.

Over the years, we have provided a wide range of atomic data needed for modelling purposes, such as cross sections for photoionisation / radiative recombination (see, e.g. Badnell 2006 ), dielectronic recombination (see, e.g. Badnell et al. 2003 [Paper I] and Abdel-Naby et al. 2012 [last paper]), ionic excitation by electron impact, as well as radiative data. While accurate atomic structure calculations remain a challenge, they can usually be carried-out to the point where uncertainties in a complex collision problem, such as electron-impact excitation of positive ions, dominate the final answer. It is not uncommon to find in the literature values that are in error by a factor of two or more. We therefore focus this review on such calculations.

Our atomic data are widely used as are included in all the main databases for astrophysical spectroscopy. For example, CHIANTI (Dere et al., 1997; Del Zanna, Dere, Young, Landi and Mason, 2015), ATOMDB (Smith et al., 2001), Cloudy (Ferland et al., 2013), MOCASSIN (Ercolano et al., 2008), XSTAR (Bautista and Kallman, 2001), PINTofALE (Kashyap and Drake, 2000), and SPEX (Mao and Kaastra, 2016). These data are used to analyse the wealth of XUV spectra of solar (SOHO, Hinode) and astrophysical (Chandra, XMMNewton, HST, FUSE) plasmas of many sources (e.g. the solar corona, stellar atmospheres, supernova remnants, AGNs, comets). Our data are also incorporated into ADAS and made available through OPEN-ADAS (open-adas.ac.uk). ADAS is widely used by the magnetic fusion community, but it is also used in solar physics. For example, APAP data were recently included in the modelling which provided a new estimate on $\mathrm{He}$ enhancement factors for the solar atmosphere (Giunta et al., 2015).

We briefly discuss the various methods used for the calculations, then mention various issues we have

‡www.apap-network.org uncovered. We then review our work on the ionic excitation by electron impact for the main isoelectronic sequences, and mention other work we have carried out.

\section{Methods and issues uncovered}

For most of our calculations, we have used the AUTOSTRuCTuRE (AS) program (Badnell, 2011) which constructs target wavefunctions using radial wavefunctions calculated in a scaled Thomas-Fermi-Dirac statistical model potential with a set of variational scaling parameters. For the configuration-interaction (CI) expansion we usually include a large number of configurations. The scaling parameters are chosen using various strategies, see e.g. Liang and Badnell (2011); Fernández-Menchero et al. (2014a). The wavefunctions are then used to calculate the radiative data (oscillator strengths and A-values) and for the scattering calculation.

For complex ions, we sometimes improve the calculations with the term energy correction (TEC) method, introduced by Zeippen et al. (1977) and Nussbaumer and Storey (1978). Within this method we add a non-diagonal correction $\boldsymbol{X}^{-1} \boldsymbol{\Delta} \boldsymbol{X}$ to the Breit-Pauli Hamiltonian matrix, where $\boldsymbol{X}$ diagonalizes the (uncorrected) $L S$ Hamiltonian and $\boldsymbol{\Delta}$ is a diagonal matrix of energy corrections. The implementation of the TEC is not trivial since it depends on the availability of experimental energies, which often have to be re-assessed. Given that only few values are normally available, we often obtain a set of 'best guess' energies $E_{\text {best }}$ by linear interpolation of the target energies (see, e.g. Del Zanna et al. 2012a).

For the scattering calculations we normally use the $R$-matrix method, using a suite of codes that we maintain and that originated from a large-scale collaboration over a long period of time. The methods are described in Hummer et al. (1993) and Berrington et al. (1995). We usually perform the calculation in the $R$-matrix inner region in $L S$-coupling including both mass and Darwin relativistic energy corrections. For the outer region calculation we then use the intermediate-coupling frame transformation method (ICFT), described by Griffin et al. (1998), which makes use of multi-channel quantum defect theory. This method is computationally much faster than other ones such as the Breit-Pauli $R$-matrix method (BPRM) while being just as accurate in practice for ions of astrophysical interest. For example, our sequence calculations cover elements from $\mathbf{H}$ to $\mathrm{Zn}$.

For the close-coupling (CC) expansion of the complex ions, sometimes we retain a smaller set of $L S$ terms. The electron exchange and non-exchange calculations are run separately. The resonance region is calculated with a fine energy grid, while a coarse 
energy mesh is chosen above all resonances. Collision strengths are 'topped-up' to infinite partial waves following Burgess (1974); Badnell and Griffin (2001). Finally, the collision strengths are extended to high energies by interpolation using the appropriate highenergy limits in the Burgess and Tully (1992) scaled domain. The high-energy limits are calculated with Autostructure following Burgess et al. (1997) and Chidichimo et al. (2003). We store the collision strengths and have developed methods to reduce their energy resolution to a coarser mesh and calculate the temperature-dependent effective collisions strengths $\Upsilon(i-j)$ (the rates) assuming Maxwellian, $\kappa$ and other electron distributions.

We (NRB) have also recently developed a new Breit-Pauli distorted wave (DW) method which bears some similarities with the historic and widely-used UCL-DW code (Eissner, 1998), but is fundamentally different. The continuum distorted waves are calculated using the same form for the distorting potential as specified for the target, but now for the $(\mathrm{N}+1)$-electron problem. The continuum orbitals are not orthogonalized to the bound orbitals, and the appropriate exchange overlap integrals are determined instead. The electrostatic and, optionally, twobody non-fine-structure $(\mathrm{N}+1)$-electron interaction Hamiltonian for the collision problem is determined in an unmixed LS-coupling representation. It is then transformed to an LSJ representation. The full $(\mathrm{N}+1)$ electron interaction Hamiltonian is transformed to a full Breit-Pauli jK-coupling representation in the same way as it is done for the inner-region Breit-Paul Rmatrix, and with the option to include two-body finestructure. Both non-relativistic and (kappa-averaged) relativistic orbitals can be used.

The AS DW runs are extremely fast and are useful to quickly calculate collision strengths where the effects of the resonances are not too large, such as transitions to high-lying levels (see, e.g. the Fe VIII and Fe IX calculations in O'Dwyer et al. 2012). Full details of this recent development (AS DW) are found in Badnell (2011).

The enhancement due to resonances in the electron-impact excitation problem could in principle be included perturbatively as well since it is the complement of dielectronic recombination, which has long been the mainstay of AS. However, early work (Badnell et al., 1993) showed significant differences between such a perturbative treatment and an $R$-matrix one. Such an approach works well for dielectronic recombination because of the weak coupling of the electron to the radiation field. A recent comparison by Fernández-Menchero, Giunta, Del Zanna and Badnell (2015) for $\mathrm{Fe}^{20+}$ between equivalent-sized $R$-matrix and earlier DW-plus-resonances calculations of Landi and $\mathrm{Gu}$ (2006) shows a significant systematic underestimate by the perturbative results. Thus, $R$-matrix calculations should be carried-out whenever possible.

\subsection{ICFT vs. Breit-Pauli R-matrix}

Despite various claims found in the literature, we would like to stress here that the ICFT approach is accurate compared to the full BPRM method, with differences at most within a few percent. Such differences are well below those typically encountered when varying all the parameters that are involved in such complex calculations.

Satisfactory comparisons between ICFT and BPRM for ions of nuclear charge up to nickel have been published. Griffin et al. (1998) studied Mg-like ions and Badnell and Griffin (1999) Ni v. Comparisons between ICFT and DARC (Dirac $R$-matrix code, developed by P. H. Norrington and I. P. Grant) were published in Liang and Badnell (2010); Liang et al. (2009a). Badnell and Ballance (2014) compared the results of ICFT, BPRM and DARC on FeIII.

Despite this, further claims on the unreliability of the ICFT approach (compared to the relativistic DARC) were recently published by Aggarwal and Keenan in a series of papers. In particular, Aggarwal and Keenan (2014) were critical about our previous work on Fe XIV (Liang et al., 2010) and Aggarwal and Keenan (2015) on our ICFT calculations for all the ions in the Be-like sequence (Fernández-Menchero et al., 2014a). Aggarwal and Keenan found large differences, with the ICFT results providing collision strengths consistently larger.

We had to run several new ICFT and BPRM calculations to show that the differences pointed out by Aggarwal and Keenan arise mainly because of the different CC and CI expansions. The significant increases in the effective collision strengths obtained with the ICFT method were mainly due to the extra resonances that are present because of the larger target expansion. Details are presented in FernándezMenchero, Del Zanna and Badnell (2015c) and Del Zanna, Liang, Badnell, Fernández-Menchero, Liang, Mason and Storey (2015), where we discuss the effects caused by the various parameters (CC and CI expansions, energy resolution, threshold position, etc).

Aggarwal and Keenan (2014) refer to a problem in an ICFT calculation of $\mathrm{O}^{2+}$ encountered by Storey et al. (2014). It should be stressed that this problem was peculiar to low-charge ions such as $\mathrm{O}^{2+}$ when resonance effective quantum numbers become small. Storey et al. (2014) described a solution such that good agreement between the ICFT and BPRM methods could be obtained even in this case. ICFT calculations for intermediate and high-charge ions are not affected by this problem. 


\subsection{DW and cascading from higher levels}

The soft X-rays $(50-170 \AA)$ are dominated by transitions from $n=4$ levels of iron ions. Atomic data for these iron soft X-ray lines were not available, so we have carried out several calculations, for Fe VIII (Del Zanna and Badnell, 2014), Fe Ix (Del Zanna, Storey, Badnell and Mason, 2014), Fex (Del Zanna et al., 2012a), Fexi (Del Zanna and Storey, 2013), FexII (Del Zanna et al., 2012b), and FexiII (Del Zanna and Storey, 2012).

We have encountered several surprising issues. First, comparisons between ICFT $R$-matrix and DW showed that for many cases (in particular for the $3 \mathrm{~s}^{2} 3 \mathrm{p}^{q} 4 \mathrm{~s}$ levels) the DW calculations underestimate significantly the (effective) collision strengths. For example, the $3 \mathrm{~s}^{2} 3 \mathrm{p}^{4} 4 \mathrm{~s}$ levels in Fex, which produce strong decays in the soft X-rays, identified by Edlén in 1936 and led to the famous discovery of the coronal forbidden line of $\mathrm{Fex}$.

Also, we found out that the cross sections for the forbidden transitions exciting the $3 \mathrm{~s} 3 \mathrm{p}^{q} 4 \mathrm{~s}$ levels are quite strong; they produce dipole-allowed decays that are stronger than those from the $3 \mathrm{~s}^{2} 3 \mathrm{p}^{q} 4 \mathrm{~s}$ levels. For example, in the Fex case, the $3 \mathrm{~s} 3 \mathrm{p}^{6}{ }^{2} \mathrm{~S}_{1 / 2}-3 \mathrm{~s} 3 \mathrm{p}^{5}$ $4 \mathrm{~s}^{2} \mathrm{P}_{3 / 2}$ transition. Del Zanna (2012) identified such decays for the coronal iron ions.

The combined effect of extra cascading and increased excitation resulted in significantly different level populations of the lower levels, including those of the ground configuration for several ions. The effects are subtle, in that collision strengths due to the larger target are typically increased by only $10 \%$ or so. However, the combined effect of a large number of higher levels cascading down increases the population of the lower levels.

\subsection{A problem with DW non-unitarized calculations}

Comparisons between the background $R$-matrix collision strengths with those obtained with the AS DW code (using the same target) are normally very satisfactory (see, e.g. Liang and Badnell 2011; Del Zanna et al. 2012a). However, we found large discrepancies (factors $\sim 10$ or more) in the collision strengths for $J-J^{\prime}=0-0$ transitions in Mg-like ions such as $3 \mathrm{~s}^{2}{ }^{1} \mathrm{~S}_{0}-3 \mathrm{p}^{2}{ }^{1} \mathrm{~S}_{0}$ (Fernández-Menchero et al., 2014b). The same problems were found in the results obtained with the Flexible Atomic Code (FAC) (Gu, 2003) by Landi (2011) for FexV. On the other hand, the values obtained with the UCL-DW code (Eissner, 1998) by Christensen et al. (1985) were in good agreement with the background $R$-matrix.

Given that a significant amount of atomic databases still use results obtained with the DW approximation, this issue deserved further investigations.
The issue, resolved in Fernández-Menchero, Del Zanna and Badnell (2015b), was related to the neglect of coupling in the scattering equations. The $R$-matrix method solves the closely-coupled scattering equations for the colliding electron and calculate the elements of the reactance matrix $K$, which is related to the transmission matrix $T$ via

$T=\frac{-2 \mathrm{i} K}{1-\mathrm{i} K}$.

The resulting scattering matrix, $S=1-T$, is unitary. The collision strength $\left(\Omega_{i j}\right)$ for any transition $i-j$ is

$\Omega_{i j} \propto\left|T_{i j}\right|^{2}$.

A significant advantage of the DW method is that it does not need to calculate the entire $K$-matrix since it solves uncoupled scattering equations. Formally, it can make use of

$T=\frac{-2 \mathrm{i} K}{(1-\mathrm{i} K)} \times \frac{(1+\mathrm{i} K)}{(1+\mathrm{i} K)}=\frac{-2 \mathrm{i} K+2 K^{2}}{1+K^{2}} \approx-2 \mathrm{i} K$,

in which case the DW method is called non-unitarized. AS DW, FAC and also HULLAC (Bar-Shalom et al., 1988) are all non-unitarized methods which neglect such coupling.

An option in the AS DW code to treating all coupling of the scattering equations as a perturbation was implemented: AS UDW. This option converts the full reactance $K$-matrices to the transmission $T$ matrices, giving rise to unitary scattering $S$-matrices. The results of the AS UDW are in good agreement with the background $R$-matrix collision strengths.

The neglect of coupling would have affected the atomic data for a few weak optically forbidden transitions in other isoelectronic sequences, calculated with non-unitarized DW codes such as FAC and HULLAC.

\subsection{R-matrix and TECs}

The calculations of more complex ions are often affected by strong mixing between levels. The mixing between fine-structure levels depends critically on their relative energy separation, and not that relative to the ground, say. Such mixing changes substantially depending on the configurations included in the target representation, particularly when the levels belong to terms arising from different configurations.

We have often encountered published calculations where target energies were close to the experimental ones, but the relative level energies were not, so incorrect oscillator strengths and collision strengths were obtained for those highly-mixed levels. For example, FexI has three $n=3, J=1$ levels, which produce amongst some of the strongest lines for this ion. Previous calculations for transitions to these levels were incorrect, and was only with an ad-hoc target 
that we were able to obtain good agreement with observations (Del Zanna et al., 2010).

Similar issues are very common in complex ions. For example, collision strengths for Fe VIII have been notoriously difficult to calculate accurately (Del Zanna, 2009). Tayal and Zatsarinny (2011) recently produced a large-scale calculation for this ion with absolute energies quite close to the experimental ones, but the spin-orbit mixing in several important transitions was incorrect, as shown in Del Zanna and Badnell (2014).

To partially resolve this long-standing issue, we have developed a new method within the ICFT $R$ matrix codes. The target Breit-Pauli mixing of the scattering/reactance matrices is introduced through the use of the TEC after algebraic $j K$-recoupling (Saraph, 1972). We note that applying TECs to the $L S$ Hamiltonian alone is equivalent to adjusting the target $L S$ eigenenergies but it does not change the eigenvectors. The resulting collision strengths for Fe VIII were shown by Del Zanna and Badnell (2014) to substantially improve agreement with observations.

\subsection{Non-Maxwellian electron distributions}

Departures from a Maxwellian distribution are expected to arise during the impulsive phase of solar flares, as is indeed observed in the hard X-rays. However, establishing the distribution is a non-trivial issue. There are various other cases in the solar corona where departures from a Maxwellian distribution have been suggested. However, accurate atomic data and modelling are required. Dufton et al. (1984) and subsequent authors (e.g. Keenan et al. (1989)) found anomalously high intensities in a high-excitation line from Si III and suggested that these discrepancies were caused by the presence of non-Maxwellian electron distributions. Our calculations and modelling showed that the Si III line intensities were actually consistent (with one exception) with Maxwellian distributions (Del Zanna, Fernández-Menchero and Badnell, 2015).

Having said that, there are many instances where simple equilibrium models of the solar transition region fail to explain observations. For example, the strong lines from Li- (C IV) and Na-like (e.g. Si IV) ions. We used our B-like cross-sections to show that strong Si IV vs. O IV enhancements could in principle be caused by non-Maxwellian distributions (Dudík, Del Zanna, Dzifčáková, Mason and Golub, 2014).

There have been searches of signatures of nonMaxwellian distributions in the solar corona but results have been inconclusive. We used the cross-sections we have calculated for the coronal iron ions to search for the best diagnostics, and pointed out which line ratios are the best to use (Dudík, Del Zanna, Mason and Dzifč́ková, 2014). We used these results and solar observations of a transient loop, to find evidence of departures from a Maxwellian distribution (Dudík et al., 2015).

\section{Excitation by electron impact}

\subsection{He-like ions}

Spectral emission lines of He-like ions have been used for diagnostics of astrophysical plasmas in the X-rays for a long time, following the seminal work of Gabriel and Jordan (1969). However, it is only recently that we have made various improvements to the atomic data.

In Whiteford et al. (2001) we performed radiationdamped $R$-matrix ICFT calculations for all the Helike ions. The target included all 49 levels up to 1s $5 l$. Several other calculations for these ions exist, however they were mostly either DW (no resonance enhancement) or did not include radiation damping (e.g. a series of papers from Aggarwal \& Keenan, see Aggarwal and Keenan 2012), which is an important effect for H- and He-like ions, as discussed e.g. in Gorczyca and Badnell (1996); Griffin and Ballance (2009).

The Zhang and Sampson (1987) collision strengths for the He-like ions have been widely used (for example within the CHIANTI database until version 8, Del Zanna, Dere, Young, Landi and Mason 2015). Zhang and Sampson (1987) calculated the contributions to the resonance enhancement associated with the $1 s 3 l^{\prime \prime} n^{\prime} l^{\prime \prime \prime}$ autoionizing levels of the Lilike ions. However, as Figure 1 shows, their calculations still underestimate the rate for the important forbidden transition. As already pointed out by Zhang and Sampson (1987), the resonances associated with the $1 s \quad 2 l^{\prime \prime}$ $n l^{\prime \prime \prime}$ autoionizing levels ( $n=2$ resonances), not included in their calculations, do provide a significant enhancement for the forbidden transition in several ions. Zhang and Sampson (1987) showed that by including estimates of the $n=2$ resonances for $\mathrm{O}$ VII and $\mathrm{Mg}$ XI (cf their Tables 3,4), better agreement was found with $R$ matrix calculations for these ions, so it is likely that the difference shown in Figure 1 is due to these resonances.

Atomic data for highly-excited levels are also needed for astrophysics. Lines from levels up to $n=10$ have been observed in laboratory plasma and recently also in X-ray spectra of solar flares (see, e.g. Kepa et al. 2006). We have recently addressed this issue (Fernández-Menchero, Del Zanna and Badnell, 2015a) with various calculations, showing that relatively accurate results can be obtained by extrapolation. 


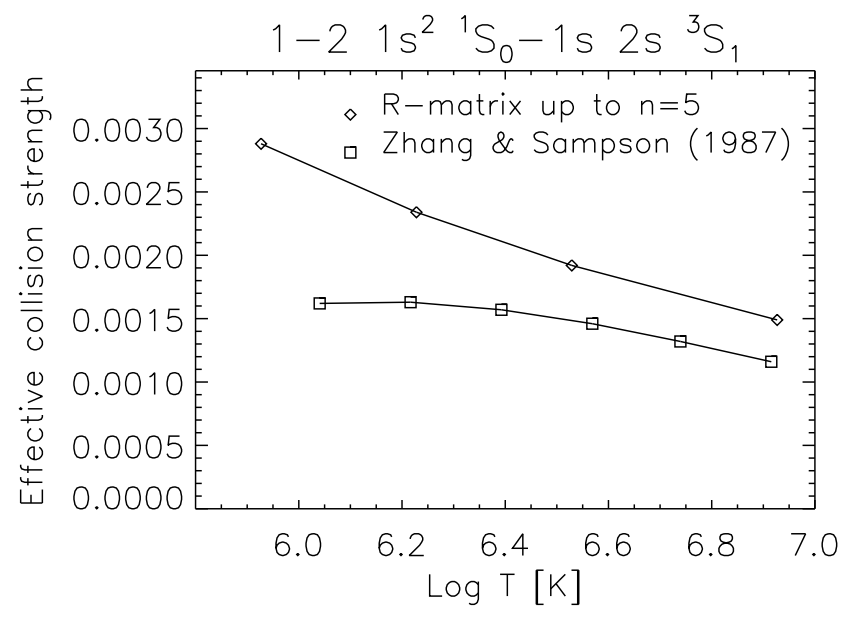

Figure 1. The rate for the forbidden line in Si XIII as calculated with the $R$-matrix ICFT codes by Whiteford et al. (2001) and by Zhang and Sampson (1987).

\subsection{Li-like ions}

We (Liang and Badnell, 2011) have performed scattering calculations for all Li-like ions from $\mathrm{Be}^{+}$to $\mathrm{Kr}^{33+}$ using the radiation- and Auger-damped ICFT $R$-matrix approach. The target included 204 closecoupling levels, with valence (up to $n=5$ ), and coreelectron excitations (up to $n=4$ ). Most of previous scattering calculations contained only 15 or 40 levels, calculated mostly with DW codes.

\subsection{Be-like ions}

Lines from Be-like ions have been used for a long time to measure electron densities (see, e.g. Munro et al. 1971 and citations) and temperatures. In particular, the intensity ratios of the resonance versus the intercombination transitions in the Belike ions is an excellent temperature diagnostic (see, e.g. Landi et al. 2001 and references). The ratio between the $2 \mathrm{~s} 2 \mathrm{p}{ }^{1} \mathrm{P}_{1}-2 \mathrm{p}^{2}{ }^{1} \mathrm{D}_{2}$ and the intercombination transition is also a good diagnostic.

Only some atomic calculations for ions in this sequence were available, and for many ions the rates were interpolated (see e.g. Keenan et al. 1986) using calculations with much more limited targets (Berrington et al., 1985). We performed the first full $R$-matrix calculation for $\mathrm{Mg}^{8+}$ (Del Zanna et al., 2008), and compared the intensities of the main lines with those obtained with the interpolated values. Significant (up to 50\%) differences were found, in some of the main lines and diagnostics. There was therefore a need for full $R$-matrix calculations for all the ions along the sequence, which we carried out in FernándezMenchero et al. (2014a). We included in the CI and $\mathrm{CC}$ expansions atomic states up to $n l=7 \mathrm{~d}$, for a total of 238 fine-structure levels and calculated atomic data for all ions from $\mathrm{B}^{+}$to $\mathrm{Zn}^{26+}$.

As previously mentioned, Aggarwal and Keenan (2015) performed DARC calculations and were critical of our results. However, as shown in FernándezMenchero, Del Zanna and Badnell (2015c) it was the Aggarwal and Keenan (2015) results that were more inaccurate because of the much smaller CI and CC expansions that they employed.

As an example of the significant differences obtained with the new data we show in Table 1 the relative intensities of the main Si XI, for a temperature and density typical of a solar active region core. The intercombination line is $50 \%$ stronger with the new data. SOHO CDS observed routinely these Si XI lines, and the values we have obtained from an active region are shown in the Table. Good agreement is found with the new data.

\subsection{B-like ions}

For the B-like ions, we have adopted the same target adopted for the FexxiI ion model by Badnell et al. (2001) to carry out for electron-impact excitation amongst 204 close-coupling levels for all ions from $\mathrm{C}^{+}$ to $\mathrm{Kr}^{31+}$ (Liang et al., 2012). For many ions, only DW data were previously available. These data are a significant improvement for many ions where only $n=2,3$ data were available. For example, the new data have significantly larger collision strengths for the $n=2$ levels, due to the extra resonances attached to all the $n=3$ levels, which were not included by Zhang et al. (1994). For the most important ions such as OIV, where previous calculations were more extended, the increased target did not produce results significantly different for the $n=2,3$ levels compared to the previous ones (Dudík, Del Zanna, Dzifčáková, Mason and Golub, 2014).

\subsection{F-like ions}

In Witthoeft et al. (2007) we presented ICFT $R$-matrix calculations for electron-impact excitation amongst $195 n=3$ close-coupling levels for all F-like ions. For several ions, rates were previously not available and were interpolated.

\subsection{Ne-like ions}

In Liang and Badnell (2010) we presented the calculations for electron-impact excitation of all Nelike ions from $\mathrm{Na}^{+}$to $\mathrm{Kr}^{26+}$ using the ICFT $R$-matrix approach, for a large target, including 209 levels up to $n=7$. For several ions (Na II, Mg III, Al IV, P VI, K X, TixiII, Crxv, Mnxvi, CoxviII and ZnXxi), atomic data were not previously available or were calculated 
Table 1. Relative intensities (photons) of some among the strongest lines for Be-like Si. They were calculated at $T=3.2 \times 10^{6} \mathrm{~K}$ and $N=3.2 \times 10^{9} \mathrm{~cm}^{-3}$ using our calculations (ICFT) and the previous Berrington et al. (1985) data.

\begin{tabular}{llcccc}
\hline \hline$i-j$ & Levels & $\begin{array}{c}\text { Int } \\
\text { ICFT }\end{array}$ & $\begin{array}{c}\text { Int } \\
\text { Previous }\end{array}$ & $\begin{array}{c}\text { Int } \\
\text { Observed (CDS) }\end{array}$ & $\lambda_{\exp }(\AA)$ \\
\hline $1-5$ & $2 \mathrm{~s}^{2}{ }^{1} \mathrm{~S}_{0}-2 \mathrm{~s} 2 \mathrm{p}{ }^{1} \mathrm{P}_{1}$ & 1.0 & 1.0 & 1.0 & 303.33 \\
$4-7$ & $2 \mathrm{~s} 2 \mathrm{p}^{3} \mathrm{P}_{2}-2 \mathrm{p}^{2}{ }^{3} \mathrm{P}_{1}$ & $9.9 \times 10^{-3}$ & $7.3 \times 10^{-3}$ & $8 \times 10^{-3}$ & 371.50 \\
$1-3$ & $2 \mathrm{~s}^{2}{ }^{1} \mathrm{~S}_{0}-2 \mathrm{~s} 2 \mathrm{p}^{3} \mathrm{P}_{1}$ & $5.9 \times 10^{-2}$ & $3.9 \times 10^{-2}$ & $5.3 \times 10^{-2}$ & 580.92 \\
$5-9$ & $2 \mathrm{~s} 2 \mathrm{p}{ }^{1} \mathrm{P}_{1}-2 \mathrm{p}^{2}{ }^{1} \mathrm{D}_{2}$ & $1.1 \times 10^{-2}$ & $8.3 \times 10^{-3}$ & $1.3 \times 10^{-2}$ & 604.12 \\
\hline
\end{tabular}

with various approximations (e.g. Si v, Ar IX, Ca XI, and Ni XIX).

\subsection{Na-like ions}

For the Na-like ions, we calculated atomic data from $\mathrm{Mg}^{+}$to $\mathrm{Kr}^{25+}$ using the ICFT $R$-matrix approach. The outer-shell calculations (Liang et al., 2009a) included in the close-coupling expansion configurations up to $n=6$. The inner-shell calculations (Liang et al., 2009b) were carried out with the ICFT $R$-matrix method with both Auger and radiation damping and represent the first such calculations. For several ions (AliII, Si Iv, Pv, S vi, Arviı,, K Ix, Cax, Tixir, Cr xiv, Mnxv, Fexvi, Coxvis, Ni xviıI, and Zn Xx), only DW data were previously available.

\subsection{Mg-like ions}

Emission lines from ions in the Mg-like sequence can be used to measure temperature and density of astrophysical plasma such as gaseous nebulae (see, e.g. Si III, Nussbaumer 1986), but excitation data for many ions in the sequence had not been calculated with any $R$-matrix or DW method. Only some ions such as Si III (see above) received special attention. We have carried out (Fernández-Menchero et al., 2014b) ICFT $R$-matrix calculations for all the ions from $\mathrm{Al}^{+}$ to $\mathrm{Zn}^{18+}$ to include a total of 283 fine-structure levels in both the CI target and CC collision expansions. These arise from the configurations $1 \mathrm{~s}^{2} 2 \mathrm{~s}^{2} \mathrm{p}^{6} 3\{\mathrm{~s}, \mathrm{p}, \mathrm{d}\} n l$ with $n=4,5$, and $l=0-4$. The Si III case was discussed in detail in Del Zanna, Fernández-Menchero and Badnell (2015).

\subsection{Al-like ions}

FexIV is one of the most important coronal ions. In Liang et al. (2010) we presented a large-scale ICFT $R$-matrix calculation which improved over the previous ones. As previously mentioned in Section 2.1, Aggarwal and Keenan (2014) were critical about our calculations, however as shown in Del Zanna, Liang, Badnell, Fernández-Menchero, Liang, Mason and Storey (2015), it was the Aggarwal and Keenan (2014) results that were more limited because of the smaller CI and CC expansions that they adopted (136 levels). The Liang et al. (2010) calculations were further improved in Del Zanna, Liang, Badnell, Fernández-Menchero, Liang, Mason and Storey (2015) by retaining the full set of 228 levels for the CC expansion.

\subsection{Si-like ions}

For FexIII, we carried out ICFT $R$-matrix calculation which included a total of 749 levels up to $n=4$ (Del Zanna and Storey, 2012). The results for the $n=3$ levels are in close agreement with those previously calculated by Storey and Zeippen (2010) while are significantly improved over the previous DW data for the $n=4$ levels. We employed a similar target for NixV (Del Zanna, Storey and Mason, 2014), finding significant differences for the important $n=3$ levels, compared to the previous DW data (Landi and Bhatia, 2012).

\subsection{P-like ions}

For Fe XII, we have carried out a large ICFT $R$-matrix calculation which included 912 levels up to $n=4$ (Del Zanna et al., 2012b). Significant differences for all the levels, including the important ones of the ground configuration, were found, as previously mentioned in Section 2.2. The changes also affected (by a factor of about 3 ) electron density measurements obtained from the $n=3$ lines and the visible forbidden lines within the ground configuration.

\subsection{S-like ions}

As in the case of FexiI, the large scale calculations for Fe XI have provided significantly different (30-50\%) results even for the $n=3$ lines, which provided the main temperature and density diagnostics for this ion. In this case, we carried out an ICFT $R$-matrix calculation which included 996 levels up to $n=4$ (Del Zanna and Storey, 2013). 


\subsection{Cl-like ions}

For Fex, we have carried out a large-scale $R$-matrix calculation (Del Zanna et al., 2012a) including 552 levels up to $n=4$. As in the previous cases, the larger model produced significantly different (30-50\%) intensities for some of the important $n=3$ lines, and also for the visible forbidden lines within the ground configuration.

\subsection{Ar-like ions}

For Fe Ix, we have carried out a large-scale ICFT $R$ matrix calculation Del Zanna, Storey, Badnell and Mason (2014) including levels up to $n=5$. In this case, only the data for a few $n=3$ transitions were significantly different, compared to those obtained with the earlier calculations by Storey et al. (2002), which included $n=2,3$ and some $n=4$ levels. On the other hand, a similar calculation we have carried out for Ni XI (Del Zanna, Storey and Badnell, 2014) showed large differences with previous $R$-matrix (Aggarwal and Keenan, 2007) and DW calculations (Bhatia and Landi, 2011).

\subsection{K-like ions}

As previously mentioned, we have carried out a large-scale ICFT $R$-matrix calculation including levels up to $n=5$ (518 levels) for Fe VIII (Del Zanna and Badnell, 2014). The new data were shown to substantially improve agreement with solar spectroscopic observations of the $n=3$ EUV lines, some of which are useful for measuring electron temperatures (Del Zanna, 2009).

\section{Atomic processes for nebular spectroscopy and modelling}

An outstanding problem in nebular astrophysics is the discrepancy between elemental abundances derived from collisionally excited lines (CEL) and from optical recombination lines (ORL). In some planetary nebulae, the discrepancy exceeds a factor of ten. One proposal to resolve this problem is that the recombination line emission originates primarily from clumps of cold $\left(10^{3} \mathrm{~K}\right)$ metal-rich material which are invisible in collisionally excited lines due to the low electron temperature (see, e.g. Liu et al. 2006).

Previous recombination theory for the most observed species (C II, O II, N II, Ne II) used calculations made in LS-coupling which included the recombination processes that are expected at conventional nebular temperatures of about $10^{4} \mathrm{~K}$. At the temperatures and densities thought to exist in the metal-rich clumps two new effects must be included: 1) the ground term fine-structure states of the recombining ion may not be statistically populated, as assumed in LS-coupling this introduces a strong dependence on electron density in the recombination line emissivities; 2) dielectronic recombination due to autoionizing states of high principal quantum number lying between the ground state fine-structure levels must be included - this process strongly affects line intensities at the low temperatures thought to exist in the clumps.

Techniques to treat the full collisional-radiative capture cascade problem including these effects have been developed (Fang et al., 2011). The new theory promises two significant advances in nebular physics. Firstly, reliable abundances can be determined from recombination lines where there is a strong dependence on the electron density and secondly, the relative intensities of recombination lines provide a new diagnostic of the conditions where the lines are emitted.

More recently it has been proposed that the CEL/ORL discrepancies could be resolved if the free electron energy distribution in nebulae is non Maxwellian (see, e.g. Nicholls et al. 2012). This suggestion is controversial since the timescales for thermalization of the free electrons have always been understood to be sufficiently short compared to the ionization/recombination times as to make departures from Maxwellian distributions very unlikely. However, while the CEL/ORL problem remains unresolved, it is important to explore all possibilities and we (Sochi and Storey, 2013; Storey and Sochi, 2013) have demonstrated a novel method based on lines originating from autoionizing states, of directly sampling the electron energy distribution at specific energies. We have also shown (Storey and Sochi, 2014) how the size of the Balmer discontinuity and the shape of the Balmer continuum can, in some nebulae, be used to examine the electron energy distribution at low energies.

The best studied CEL/ORL comparison involves the strong collisionally excited forbidden lines of $\mathrm{O}^{2+}$ and the abundant $\mathrm{O}$ II lines formed as it recombines. A coherent analysis of the problem requires firstly, accurate collision strength data for $\mathrm{O}^{2+}$, enabling reliable rate coefficients to be calculated with non-Maxwellian distributions, and secondly effective recombination coefficients for lines of $\mathrm{O}$ II and H I also calculated with non-Maxwellian distributions. We have recently completed an elaborate BreitPauli R-matrix calculation of collision strengths for electron scattering from $\mathrm{O}^{2+}$ (Storey et al., 2014) with tabulations of effective collision strengths calculated with a $\kappa$-distribution of electron energies (Storey and Sochi, 2015b), including the Maxwellian results as a limiting case. We have also computed the line intensities for the $\mathrm{H}$ I nebular recombination spectrum 
calculated with $\kappa$-distributed electron energies (Storey and Sochi, 2015a). The results were provided in the form of a code to interpolate in the three dimensions of electron density, temperature and $\kappa$.

\section{Conclusions}

The new APAP calculations have substantially improved on previous work for many isoelectronic sequences and coronal ions. Much of these data have been incorporated into the CHIANTI database version 8 (Del Zanna, Dere, Young, Landi and Mason, 2015). However, there is a clear need for similar complex calculations for other ions along many of the sequences we have worked on. There is also a clear need to complete our large-scale R-matrix calculations for other sequences. In particular, accurate atomic data are needed for the C-like, N-like and O-like sequences.

\section{Acknowledgments}

The APAP (formerly known as UK Rmax) work was funded by PPARC/STFC (UK) over the past years through the University of Strathclyde grants (1999-2002: PPA/G/S/1997/00783; 2004-2007: PPA/G/S/2003/0005;2008-2011:PP/E001254/1; 2012 2015: ST/J000892/1) and the University of Cambridge DAMTP astrophysics grant (2014-2017).

\section{References}

Abdel-Naby S A, Nikolić D, Gorczyca T W, Korista K T and Badnell N R 2012 A $\mathscr{G}$ A 537, A40.

Aggarwal K M and Keenan F P 2007 A $\& A$ 475, 393399.

Aggarwal K M and Keenan F P 2012 Phys. Scr 85(2), 025305.

Aggarwal K M and Keenan F P 2014 MNRAS 445, 2015-2027.

Aggarwal K M and Keenan F P 2015 MNRAS 447, 3849-3855.

Badnell N R 2006 ApJS 167, 334-342.

Badnell N R 2011 Computer Physics Communications 182, 1528-1535.

Badnell N R and Ballance C P 2014 ApJ 785, 99.

Badnell N R and Griffin D C 1999 Journal of Physics B Atomic Molecular Physics 32, 2267-2276.

Badnell N R and Griffin D C 2001 Journal of Physics B Atomic Molecular Physics 34, 681-697.

Badnell N R, Griffin D C, Gorczyca T W and Pindzola M S 1993 Phys. Rev. A 48, 2519.
Badnell N R, Griffin D C and Mitnik D M 2001 Journal of Physics B Atomic Molecular Physics 34, 50715085.

Badnell N R, O'Mullane M G, Summers H P, Altun Z, Bautista M A, Colgan J, Gorczyca T W, Mitnik D M, Pindzola M S and Zatsarinny O $2003 A \& A$ 406, 1151-1165.

Bar-Shalom A, Klapisch M and Oreg J 1988 38, 17731784.

Bautista M A and Kallman T R 2001 ApJS 134, 139149.

Berrington K A, Burke P G, Dufton P L and Kingston A E 1985 Atomic Data and Nuclear Data Tables 33, 195.

Berrington K A, Eissner W B and Norrington P H 1995 Computer Physics Communications 92, 290-420.

Bhatia A K and Landi E 2011 Atomic Data and Nuclear Data Tables 97, 50-108.

Burgess A 1974 Journal of Physics B Atomic Molecular Physics 7, L364-L367.

Burgess A, Chidichimo M C and Tully J A 1997 Journal of Physics B Atomic Molecular Physics 30, 33-57.

Burgess A and Tully J A 1992 A $6 A$ 254, 436-53.

Chidichimo M C, Badnell N R and Tully J A 2003 A\&A 401, 1177-1183.

Christensen R B, Norcross D W and Pradhan A K 1985 Phys. Rev. A 32, 93-104.

Del Zanna G 2009 A $\& A$ 508, 513-524.

Del Zanna G 2012 A $6 A$ 546, A97.

Del Zanna G and Badnell N R 2014 A\&A 570, A56.

Del Zanna G, Dere K P, Young P R, Landi E and Mason H E 2015 A 6 A 582, A56.

Del Zanna G, Fernández-Menchero L and Badnell N R 2015 AधA 574, A99.

Del Zanna G, Liang G Y, Badnell N R, FernándezMenchero L, Liang G Y, Mason H E and Storey P J 2015 MNRAS 454, 2909.

Del Zanna G, Rozum I and Badnell N 2008 A $\because A$ 487, 1203-1208.

Del Zanna G and Storey P J 2012 A 6 A 543, A144.

Del Zanna G and Storey P J 2013 A\&A 549, A42.

Del Zanna G, Storey P J and Badnell N R 2014 A\&A 566, A123.

Del Zanna G, Storey P J, Badnell N R and Mason H E 2012 a A\&A 541, A90.

Del Zanna G, Storey P J, Badnell N R and Mason H E $2012 b$ A $\& A$ 543, A139.

Del Zanna G, Storey P J, Badnell N R and Mason H E 2014 A\&A 565, A77. 
Del Zanna G, Storey P J and Mason H E 2010 A $\mathscr{G} A$ 514, A40+.

Del Zanna G, Storey P J and Mason H E 2014 A $G A$ 567, A18.

Dere K P, Landi E, Mason H E, Monsignori Fossi B C and Young P R 1997 A $\& A S$ 125, 149-173.

Dudík J, Del Zanna G, Dzifčáková E, Mason H E and Golub L 2014 ApJ 780, L12.

Dudík J, Del Zanna G, Mason H E and Dzifčáková E 2014 A\&A 570, A124.

Dudík J, Mackovjak Š, Dzifč́áková E, Del Zanna G, Williams D R, Karlický M, Mason H E, Lörinčík J, Kotrč P, Fárník F and Zemanová A 2015 ApJ 807, 123.

Dufton P L, Kingston A E and Keenan F P 1984 ApJ 280, L35-L37.

Eissner W 1998 Computer Physics Communications 114, 295-341.

Ercolano B, Young P R, Drake J J and Raymond J C 2008 ApJS 175, 534-542.

Fang X, Storey P J and Liu X W 2011 A $6 A$ 530, A18.

Ferland G J, Porter R L, van Hoof P A M, Williams R J R, Abel N P, Lykins M L, Shaw G, Henney W J and Stancil P C 2013 RMXAA 49, 137-163.

Fernández-Menchero L, Del Zanna G and Badnell N R 2014a A\&A 566, A104.

Fernández-Menchero L, Del Zanna G and Badnell N R $2014 b$ A 6 A 572, A115.

Fernández-Menchero L, Del Zanna G and Badnell N R 2015 a A\&A p. submitted.

Fernández-Menchero L, Del Zanna G and Badnell N R $2015 b$ A $ॄ$ A 577, A95.

Fernández-Menchero L, Del Zanna G and Badnell N R 2015c MNRAS 450, 4174-4183.

Fernández-Menchero L, Giunta A, Del Zanna G and Badnell N R 2015 Journal of Physics B Atomic Molecular Physics p. submitted.

Gabriel A H and Jordan C 1969 MNRAS 145, 241-+.

Giunta A S, Fludra A, Lanzafame A C, O'Mullane M G, Summers H P and Curdt W 2015 ApJ 803, 66.

Gorczyca T W and Badnell N R 1996 Journal of Physics B Atomic Molecular Physics 29, L283-L290.

Griffin D C, Badnell N R and Pindzola M S 1998 Journal of Physics B Atomic Molecular Physics 31, 3713-3727.

Griffin D C and Ballance C P 2009 Journal of Physics B Atomic Molecular Physics 42(23), 235201.

Gu M F 2003 ApJ 582, 1241-1250.

Hummer D G, Berrington K A, Eissner W, Pradhan A K, Saraph H E and Tully J A 1993 A\&A 279, 298309.
Kashyap V and Drake J J 2000 Bulletin of the Astronomical Society of India 28, 475-476.

Keenan F P, Berrington K A, Burke P G, Dufton P L and Kingston A E 1986 Phys. Scr 34, 216-220.

Keenan F P, Dufton P L, Kingston A E and Cook J W 1989 ApJ 340, 1135-1139.

Kepa A, Sylwester J, Sylwester B, Siarkowski M, Phillips K J H and Kuznetsov V D 2006 Advances in Space Research 38, 1538-1542.

Landi E 2011 97, 587-647.

Landi E and Bhatia A K 2012 Atomic Data and Nuclear Data Tables 98, 862-893.

Landi E, Doron R, Feldman U and Doschek G A 2001 ApJ 556, 912-918.

Landi E and Gu M F 2006 ApJ 640, 1171-1179.

Liang G Y and Badnell N R 2010 A $\mathscr{~ A ~ 5 1 8 , ~ A 6 4 + . ~}$

Liang G Y and Badnell N R 2011 A 6 A 528, A69+.

Liang G Y, Badnell N R, Crespo López-Urrutia J R, Baumann T M, Del Zanna G, Storey P J, Tawara H and Ullrich J 2010 ApJS 190, 322-333.

Liang G Y, Badnell N R and Zhao G 2012 A\&A 547, A87.

Liang G Y, Whiteford A D and Badnell N R $2009 a$ $A \& A$ 500, 1263-1269.

Liang G Y, Whiteford A D and Badnell N R $2009 b$ Journal of Physics B Atomic Molecular Physics 42(22), 225002.

Liu X W, Barlow M J, Zhang Y, Bastin R J and Storey P J 2006 MNRAS 368, 1959-1970.

Mao J and Kaastra J 2016 A $\&$ A 587.

Munro R H, Dupree A K and Withbroe G L 1971 Sol. Phys. 19, 347-355.

Nicholls D C, Dopita M A and Sutherland R S 2012 ApJ 752, 148.

Nussbaumer H 1986 A $\& A$ 155, 205-209.

Nussbaumer H and Storey P J 1978 A $\mho A$ 64, 139-144.

O’Dwyer B, Del Zanna G, Badnell N R, Mason H E and Storey P J 2012 A $E A$ 537, A22.

Saraph H E 1972 Computer Physics Communications 3, 256-268.

Smith R K, Brickhouse N S, Liedahl D A and Raymond J C 2001 ApJ 556, L91-L95.

Sochi T and Storey P J 2013 Atomic Data and Nuclear Data Tables 99, 633-650.

Storey P J and Sochi T 2013 MNRAS 430, 599-610.

Storey P J and Sochi T 2014 MNRAS 440, 2581-2587.

Storey P J and Sochi T 2015a MNRAS 449, 2974-2979.

Storey P J and Sochi T $2015 b$ MNRAS 446, 1864-1866. 
Storey P J, Sochi T and Badnell N R 2014 MNRAS 441, 3028-3039.

Storey P J and Zeippen C J 2010 A 6 A 511, A78+.

Storey P J, Zeippen C J and Le Dourneuf M 2002 A $\mathscr{E} A$ 394, 753-762.

Tayal S S and Zatsarinny O 2011 ApJ 743, 206.

Whiteford A D, Badnell N R, Ballance C P, O'Mullane M G, Summers H P and Thomas A L 2001 Journal of Physics B Atomic Molecular Physics 34, 3179-3191.

Witthoeft M C, Whiteford A D and Badnell N R 2007 Journal of Physics B Atomic Molecular Physics 40, 2969-2993.

Zeippen C J, Seaton M J and Morton D C 1977 MNRAS 181, 527-540.

Zhang H L, Graziani M and Pradhan A K 1994 A $\mathscr{E} A$ 283, 319-330.

Zhang H and Sampson D H 1987 ApJS 63, 487-514. 